\title{
NUEVAS TECNOLOGÍAS EN LA ENSEÑANZA: ¿CONTINENTES O HERRAMIENTAS DE LA CULTURA HUMANA?
}

Hugo A. Kofman

Universidad Nacional del Litoral, Argentina

\section{UN ENFOQUE PEDAGÓGICO INICIAL}

Las nuevas tecnologías han irrumpido a ritmo vertiginoso, brindando una serie de herramientas y contextos de comunicación y de aprendizaje, de enorme potencialidad. En un extremo están los intereses comerciales, que presionan fuertemente para imponer las nuevas modas que les resultan mas lucrativas. En la otra punta están los alumnos y las instituciones educativas, considerados en este caso como el gran mercado de esas tecnologías. En el medio estamos los docentes, que asumimos posiciones diversas frente al cambio: desde las más conservadoras hasta las más innovadoras.

En cuanto al criterio de incorporación de las nuevas tecnologías, es necesario tener en cuenta que "las repercusiones de los nuevos desarrollos tecnológicos requieren ser estudiados desde una perspectiva pedagógica" (Maggio, 2000, pág 110), ya que "la transformación de las formas de enseñar no se produce por la renovación de los artefactos, sino por la reconstrucción de los encuadres pedagógicos de dicha renovación" (Idem).

Al respecto, parece haber cierto consenso en los pedagogos en reconocer el rezago de esta disciplina frente a la tecnología. Así se podrían interpretar, por ejemplo, afirmaciones como: "La modalidad a distancia necesita redefinirse a partir del aporte de los desarrollo teóricos de las ciencias sociales en general y de la didáctica en particular. Entre otros, los desarrollos en el campo de la lingüística, la sicología cognitiva y la antropología social configuran nuevos modos de entender el trabajo de los profesores y los alumnos, y generan sólidas bases para el estudio de la enseñanza y el desarrollo de propuestas pedagógicas innovadoras" (Maggio, 2000).

Quizás un punto de vista sensato frente a las nuevas tecnologías consiste en no descartar ninguna de las posibilidades que brindan, lo cual es diferente a la actitud de correr detrás de lo último. De esta última forma se correría el riesgo de desaprovechar importantes desarrollos antes que hayan demostrado sus virtudes o deficiencias. Así parece haber sucedido con la enseñanza de resolución de problemas mediante programación de algoritmos, realizada en la década del 80 , lo que proyectó a nuestro país a los primeros puestos mundiales en las olimpíadas internacionales de informática. Al respecto, hay indicios, hasta ahora no analizados sistemáticamente, de que la mayor parte de los jóvenes estudiantes secundarios que participaron en esa experiencia se convirtieron luego en investigadores de primera línea, lo cual podría representar una relación causal. Hoy esa modalidad de uso de la computadora, desapareció en la escuela pública, y en el mejor de los casos fue reemplazada por el aprendizaje de aplicación de programas utilitarios. 
De igual manera, en la primera mitad de la década de los 90 se produjo un fuerte desarrollo de las simulaciones y los sistemas computarizados de adquisición de datos, aplicados en este caso a la enseñanza presencial en trabajos prácticos de Física. Antes que esas herramientas cognitivas se hayan estudiado en profundidad y aprovechado masivamente, fueron desplazadas por nuevas herramientas que configuran el paradigma actual: Internet y Educación a Distancia.

Estos cambios se pueden percibir fácilmente analizando la evolución de las temáticas de los congresos de Informática Educativa y de los de Enseñanza de la Física. Actualmente Internet ocupa el centro de la escena en lo que hace a las nuevas tecnologías, y en particular la utilización de plataformas ELearning, cada vez más sofisticadas. En un escalón inferior se encuentran los sistemas para aprendizaje colaborativo y cooperativo a distancia.

\section{INTERNET EN LA EDUCACIÓN Y EN LA CULTURA HUMANA}

Internet pasó a ser sinónimo de nuevas tecnologías. Castells (1997) compara la actual revolución tecnológica y su impacto en el ser humano, con dos hechos singulares en la historia de la humanidad: el nacimiento del alfabeto en Grecia hace 2700 años, y la invención de la imprenta hace más de 500 años. Sostiene que "El surgimiento de un nuevo sistema de comunicación electrónico caracterizado por su alcance global, su integración de todos los medios de comunicación y su interactividad potencial, está cambiando nuestra cultura, y lo hará para siempre" (pág. 361). El carácter totalizador del mismo es tal, que: "Todos los mensajes de toda clase quedan encerrados en el medio, porque este se ha vuelto tan abarcador, tan diversificado, tan maleable, que absorbe en el mismo texto multimedia el conjunto de la experiencia humana, pasada, presente y futura" (pág 406).

Basado en esos conceptos, el autor prevé un cambio global de la cultura y de las relaciones humanas, de modo que: 'Lo que caracteriza al nuevo sistema de comunicación, basado en la integración digitalizada e interconectada de múltiples modos de comunicación, es su capacidad de incluir y abarcar todas las expresiones culturales. Por su existencia, en este tipo de sociedad, toda clase de mensajes funcionan en un modo binario: presencia/ausencia en el sistema de comunicación multimedia. Sólo la presencia de este sistema integrado permite la comunicabilidad y socialización del mensaje. Todos los demás se reducen a la imaginación individual o a las subculturas cara a cara cada vez más marginadas" (pág 407).

El cambio cultural sería tan profundo que prácticamente lo real y lo virtual se ubicarían al mismo nivel en una especie de interacción mutua, al que el autor propone designar como "virtualidad Real" (pág. 405), a diferencia de la ya clásica "realidad virtual".

Sin embargo, Castells admite que el uso de Internet no se generalizará en un futuro inmediato como medio de comunicación (pág, 391), por lo que en la sociedad se produciría un fortalecimiento de los modelo sociales ya existentes, y nuevas formas de exclusión. Esto estaría ligado al "reforzamiento de las redes sociales culturalmente dominantes, así como el aumento de su cosmopolitismo y globalización" (pág. 396). 
A pesar de esto, el autor es optimista en cuanto a las potencialidades democratizantes de este medio de comunicación, considerando que "la arquitectura de esta tecnología de red es tal, que es muy difícil censurarla o controlarla”(pág 384). Afirmación que, a cinco años de realizada, se podría poner en tela de juicio, sobre la base de la revelación de la existencia de poderosos sistemas de captura y control de mensajes que operan en los países del capitalismo central, tales como el conocido sistema "Echelon".

Por otra parte, y sin entrar por ahora en la discusión de carácter cultural (que abarca la educación), los conceptos del autor, y las situaciones que reflejan, sería importante que sean analizadas desde la óptica de países del capitalismo periférico como el nuestro, y en particular desde una realidad de más de un $50 \%$ de la población ubicado debajo de la línea de pobreza. Análisis que habría que plantear a partir de la hipótesis de que Internet podría constituirse en un elemento más de diferenciación y marginación social, en la medida en que no se desarrollen propuestas alternativas que permitan aprovechar la enorme potencialidad de ese medio en beneficio de toda la comunidad.

\section{QUE ENTENDEMOS POR TECNOCRACIA}

Burbules (2001), desde una óptica similar a la de Castells, analiza el tema Internet, al cual reduce prácticamente las NTIC en educación. Plantea, acertadamente según nuestra opinión, que las nuevas tecnologías en la medida en que se aplican masivamente se van haciendo prácticamente invisibles (pág 13).

En cuanto a su impacto en la educación, este autor discrimina cuatro posturas: la primera sería aquella que considera las NTIC como la nueva panacea para la educación. La segunda se ubicaría en un extremo opuesto, considerando a éstas como meras herramientas que permitirían hacer lo mismo que antes pero más rápido y en forma más eficiente. La tercera, a la que considera mas sensata que las dos anteriores, plantearía que las NTIC serían herramientas no neutrales, las cuales podrían producir efectos positivos y negativos sobre los usuarios, de modo que habría que utilizarlas de manera crítica. Esta última concepción, en la que se ubicaría la mayor parte de los "observadores serios" (pag 27) no limitaría el impacto solo a una cuestión de eficiencia, sino que considera que las NTIC facilitarían la formulación de nuevos objetivos en educación, a los que antes ni se había tenido en cuenta (pág 28).

Las tres concepciones antes mencionadas son caracterizadas por el autor como propias de una "mentalidad tecnocrática", a la cual imputa la suposición de que la relación entre medios y fines está predeterminada"(pág 28). Dado que el ser humano no estaría en condiciones de discriminar de antemano los distintos efectos, entonces estaría imposibilitado de tomar decisiones trascendentes para orientar el uso de las NTIC hacia lo que se defina previamente como fines más nobles.

De acuerdo con el autor, "los ordenadores nos enfrentan de continuo con la imposibilidad de separar las consecuencias deseables de las que no lo son" (pág. 30), lo cual está relacionado al concepto de que "Ios medios y fines se interpenetran y cada uno de ellos se configura a la luz del otro"(pág. 28). Sobre esta base propone pensar de una manera "postecnocrática", aceptando que las NTIC son "artificios que modifican las percepciones que las personas tienen de sí mismas como agentes, sus relaciones mutuas, 
sus interpretaciones del tiempo y de la velocidad, sus posibilidades de hacer pronósticos, etc.; en suma, todas las dimensiones del cambio en la forma de pensar sobre medios y fines, objetivos y eficacia"(pág 29).

Esta cuarta postura frente a las NTIC para la educación, propuesta por el autor como "relacional", pone su "acento en la inseparabilidad de lo bueno y lo malo en todas las circunstancias humanas complejas, y el error de imaginar que esas cuestiones pueden evaluarse fácilmente en forma individual"(pág 31). Se apoya finalmente en otra afirmación de carácter general, según la cual "cualquier cosa lo bastante poderosa como para hacer el bien o el mal en gran escala siempre es simultáneamente peligrosa". De modo que "debemos tener presente que las nuevas tecnologías son intrínsecamente peligrosas y no engañarnos creyendo que somos sus amos"(pág. 31).

La postura relacional niega entonces la posibilidad de que la sociedad pueda tomar decisiones de fondo sobre las NTIC, a causa de que esas tecnologías están cambiando de fondo todos los aspectos de la sociedad, y en definitiva no se puede saber como se irá configurando la misma. A pesar de esto, el autor realiza un análisis profundo del rol abarcador de Internet, destacando aspectos favorables y riesgos, y proponiendo pautas para su utilización.

De hecho, entonces, asume una posición que consiste en considerar que Internet se está convirtiendo en el medio cada vez mas abarcador de las comunicaciones humanas, y que la educación del futuro se realizará fundamentalmente en ese contexto. Destaca en esto a la formación de nuevas comunidades educativas a través de Internet, y al hecho de que este medio "ha dado un tremendo estímulo a la tendencia a impartir la enseñanza en el hogar" (pág. 290).

Estos conceptos sirven de fundamento a posturas frente a la educación como la que tienen muchas instituciones que se dedican a la educación a distancia, tal como el Instituto Tecnológico de Monterrey, en el sentido de que "la educación a distancia será la piedra angular de la educación del siglo XXI" (Villaseñor Sánchez, 2000)

Antes de abocarnos al análisis de los conceptos de fondo expresados por Burbules, convendría detenernos en la argumentación que el autor utiliza para criticar las otras posturas. Cuando habla del "error de imaginar que esas cuestiones pueden evaluarse fácilmente en forma individual”, en realidad no está refutando nada, porque la discusión no está planteada en términos de facilidad o dificultad, sino en el hecho de si es posible o no realizar tal evaluación. Aunque sea difícil.

Por otra parte, la calificación de tecnocráticas a las otras posturas no parece condecirse con el término utilizado, al menos para la segunda y la tercera de las mismas: tecnocracia es un término que proviene de la política y está asociado a una forma de gobierno formado por especialistas que toman sus decisiones sobre la base exclusiva de la eficiencia. En ese paradigma, las otras dimensiones del ser humano y de la sociedad (cultura, ética, filosofía, concepto de calidad de vida, etc.) quedan relegadas y subordinadas a los imperativos de la eficiencia. Es el dominio de la técnica por sobre todos los aspectos del ser humano. No parece ser ésta la postura llamada de la "herramienta no neutral", porque la misma (correcta o incorrectamente) entroniza al ser humano por sobre la técnica. Inclusive se podría considerar 
que la postura de Burbules estaría mas cerca del concepto tecnocrático, en la medida en que concibe la técnica como un ente poderoso con vida propia, frente al cual los seres humanos no podemos siquiera diferenciar sus aspectos positivos y negativos, y seremos trasformados irreversiblemente por la misma.

\section{¿HABRÁ UNA ALTERNATIVA HUMANISTA SERIA AL POSITIVISMO REINANTE?}

Es verdad que las revoluciones tecnológicas han producido siempre cambios profundos e impredecibles en la sociedad, en la cultura y en el ser humano a nivel individual. $A$ tal punto que las nuevas ideas de los siglos XIX y XX es posible que hayan sido más el producto de la gran revolución industrial que de la brillantez de los grandes pensadores. Éstos desarrollaron sus ideas gracias al nuevo contexto socio económico que se desplegaba simultáneamente. Pero el fenómeno no fue un hecho mecánico y en una sola dirección. Hubo quienes, con sus ideas, favorecian un modelo de utilización de la tecnología que favorecía los intereses de la pujante clase capitalista de la época; pero había otros que denunciaban sus lados oscuros y proponían que los trabajadores constituían una clase que podía plantear un modelo de sociedad en el que las grandes riquezas producidas se distribuyeran de forma más equitativa y sin destruir el medio ambiente. En medio de una poderosa revolución industrial se desarrollaban corrientes de pensamiento diametralmente opuestas.

No hay razón para que hoy, en los marcos de la gran revolución de las NTIC, no se desarrollen distintas corrientes de pensamiento, las que a su vez respondan a intereses diversos, y por lo tanto a diferentes concepciones sobre el ser humano. Si bien es cierto que nadie puede predecir los cambios que las NTIC producirán en la sociedad, resulta discutible decretar la imposibilidad de abordar tal discusión. No aceptarla equivaldría a asumir la posición actualmente dominante, en este caso desde una argumentación positivista.

Efectivamente, en el pensamiento positivista "todos los hechos son singulares e individuales, no [se] busca comprender, sólo describir lo sucedido en un orden inalterable y sin conexión ni relación entre los hechos de la política, la economía, la sociedad y las manifestaciones culturales.

Todo aparece atomizado, desconectado. El conocimiento es absolutizado y no permite la interdisciplinariedad al presentar la realidad como una enunciación taxativa de hechos y cosas". (Maglio, 1998)

Esta visión atomizada, que hace énfasis en la descripción "objetiva" de "lo que ocurre", sin posibilidad de realizar un análisis desde una visión amplia de las ciencias sociales y humanas se puede emparentar con la "imposibilidad de separar las consecuencias deseables de las que no lo son"(Burbules, 2000, pag. 30). Positivismo que, de hecho, niega la posibilidad de que el ser humano juegue un rol transformador en la sociedad a través de sus decisiones concientes.

De ser así, tampoco podrían plantearse temas de discusión desde posiciones éticas, sociales y antropológicas, tales cómo la clonación humana reproductiva, la fabricación de armas de destrucción masiva cada vez más potentes, la destrucción del medio ambiente por la economía de desarrollo infinito, etc. Dado que el desarrollo tecnológico empuja ese proceso, sería absurdo oponerse al mismo. 
Decíamos que no hay razón para que no aparezcan posturas filosóficas distintas frente a las NTIC; y de hecho existen. Una de ellas es la formulada por Humberto Maturana (2000), quien desde un pensamiento sistémico y una filosofía humanista, plantea que es la sociedad, democráticamente organizada, la que debe tomar las decisiones sobre la forma en que se usarán las NTIC. Que no todo lo que se puede investigar o desarrollar haya que hacerlo necesariamente. Es decir, que la investigación es inseparable de sus fines, y son éstos los que hay que someter previamente a discusión (Maturana, 2000).

A diferencia de los autores antes citados, el autor chileno plantea, por ejemplo, que hay muchos aspectos de las relaciones humanas en que la cercanía corporal es insustituible. De modo que Internet no tendría la "capacidad de incluir y abarcar todas las expresiones culturales" tal como propone Castells, ni el "cara a cara" de las relaciones humanas estaría prácticamente destinado a extinguirse por imperio de un determinismo tecnológico. En todo caso, es la sociedad, y no la tecnología, la que debería decidir al respecto.

Maturana realiza una afirmación fuerte respecto a la importancia de las emociones en la educación, en relación con los contenidos:

"la educación ha seguido el camino de los contenidos, bajo el supuesto de que es un instrumento para asegurar ciertas formas de quehacer. Con ello ha desaparecido el aspecto fundamental de la educación que es la incorporación de los niños a un modo de convivencia que los adultos queremos, desde un punto de vista humano. Ahora, si a lo que aspiramos es que los niños sean simplemente instrumentos de producción, por supuesto que el camino que ha seguido la educación es consecuente. Pero si lo que queremos es que los niños crezcan como ciudadanos, como seres éticos, responsables, que posean un vivir digno, estético, grato, creativo en el sentido de que tengan imaginación, que tengan presencia para el bienestar, hay que preocuparse de las emociones"1.

En ese mismo sentido, el pensador Chileno considera que:

"Lo central de la educación es la formación humana. El que nuestros niños crezcan como seres que se respeten a si mismos y respeten a los demás, y que puedan decir que si o que no desde si. El respeto no es la obediencia, el respeto es la posibilidad de colaborar.

Pero para que esto pase en nuestras escuelas, nuestros profesores tienen que respetarse a si mismos, tienen que actuar desde si en la confianza de que ellos son el recurso fundamental de la educación; no los computadores, no la conexión a Internet, pues estos son sólo instrumentos.

En tanto los profesores se respeten a si mismos van a poder generar espacios en los cuales los niños podrán aprender cualquier cosa, lo que sea. Ni las matemáticas, ni la física, ni la química, ofrecen ninguna dificultad en sí. Las dificultades están en la emoción, en el miedo, en la ambición, en las expectativas que los demás tienen sobre uno y que uno tiene que satisfacer.

1 (http://www.mdnh.org/educacion/educadores.html\#4_3. Acceso: Junio de 2002). 
Lo que necesitamos es que los niños aprendan a ser lo que quieren ser y adquieran el bagaje de elementos necesarios para poder orientarse de la manera que quieran, como seres sociales, responsables, en cualquier mundo que les toque vivir. ¡No tenemos idea cómo va a ser el siglo XXI! " (Maturana, 1997).

Estos conceptos abonan la posibilidad de un enfoque distinto al propuesto por Castells y Burbules en relación a las NTIC en la enseñanza. Es la sociedad la que pre-determina los objetivos de la educación, y en función de éstos decide sobre la modalidad de utilización de las tecnologías. Si bien es cierto que éstas brindan nuevas posibilidades y permiten establecer nuevos objetivos, es en definitiva la sociedad y no las tecnologías la que define cuales serán los objetivos de la educación. Ya sea para avanzar hacia una sociedad mas humanizada y equitativa, o para consolidar la tendencia de la actual sociedad de la opulencia y la miseria extrema.

De hecho que la discusión que se requiere no es sencilla. Abarca temas supuestamente triviales como: si la posibilidad de acceso masivo a la tecnología hará obsoleta la enseñanza de la suma mental de números de un solo dígito, otros cómo el análisis de los factores emocionales en el marco de la enseñanza a distancia, hasta la posibilidad de que en esta modalidad se establezcan contenidos rígidos y absolutamente controlados por los organismos del poder. Estos y muchos otros aspectos debieran configurar la agenda del debate pedagógico y político sobre las NTIC en la enseñanza.

La discusión sobre si es conveniente mantener la educación en ámbitos escolares presenciales o trasladarla al hogar no parece una cuestión trivial, ni que convenga dejarla al devenir de los acontecimientos o a las modas tecnológicas. Se trata de definiciones pedagógicas que tienen que ver no solo con criterios de eficiencia, sino de los objetivos que se planteen para la educación. En particular, respecto a sus componentes emocionales y a los valores que la misma debe contribuir a formar. La convivencia entre estudiantes no es una cuestión menor respecto a estas dimensiones de la educación, y en ese aspecto no es lo mismo la comunicación directa que la que se mediatiza a través de los sistemas digitalizados. Lo mismo habría que considerar en cuanto a la relación entre profesor y alumno. En fin, temas que atañen a la pedagogía, la sicología, la sociología y la antropología.

En particular, convendría también tener en cuenta ciertas contribuciones que ha hecho la Programación Neurolingüística (PNL) en lo que hace a la comunicación humana; cuestiones que pueden rescatarse desde sus aportes empíricos, sin necesidad de suscribir el trasfondo conductista de esta corriente. Es así que la misma plantea que "si las palabras son el contenido del mensaje, las posturas, gestos, expresión y tono de voz son el contexto en el que el mensaje está enmarcado, y juntos dan sentido a la comunicación" O'Connor et al, 1996, pag. 48). En tal sentido, el autor se refiere a investigaciones que dan cuenta del escaso peso que tienen las palabras frente al lenguaje corporal y al tono de voz en la comunicación entre las personas. Estos conceptos podrían fortalecer la importancia de la comunicación directa, no mediatizada, que está involucrada en la enseñanza presencial. 


\section{¿TIENE VIGENCIA EL ENFOQUE ASOCIATIVO?}

Varios autores, como Pea (1993), Salomón (1993) y otros, han desarrollado el concepto de cogniciones o inteligencias distribuidas, basado en la asociación entre el ser humano y el entorno social, material y simbólico con el que desarrolla su actividad intelectual. Es así que se producen asociaciones entre personas y entre personas y máquinas como las computadoras, de manera tal que se potencian las funciones cognitivas. Esto representa una delegación del hombre a la máquina de aquellas tareas tediosas o inalcanzables mental o manualmente, lo cual le aumenta la libertad para ocuparse de aquellas funciones de mayor nivel cognitivo. Pero implica mucho más que eso: la asociación entre muchas personas y las posibilidades de miles de máquinas trabajando en redes, significa una manera de conducir el pensamiento cualitativamente diferente a la del pensador solitario, aun de aquel que se ayuda con una máquina.

Sin embargo, estos cambios no le hacen perder al ser humano su posición de centralidad en esos sistemas, su mente no queda diluida en la red. $Y$ así como la regla y el compás, como herramientas cognitivas, permitieron a los griegos desarrollar la geometría euclidiana, que significó un inmenso logro para la humanidad, la computadora e Internet puede facilitar al ser humano la adquisición de conocimientos significativamente mas elevados. Pero la esencia de la relación entre hombre y herramienta no cambia.

Pero habrá que admitir que las herramientas actuales, cada vez mas potentes, en la medida que no sean puestas concientemente al servicio de toda la sociedad, pueden llegar a constituirse -quizás ya lo sean- en poderosos medios de dominación y de exclusión de las grandes mayorías por parte de una minoría privilegiada que las domina. El rechazo a tal posibilidad, nos debe llevar, precisamente, no a rechazar la tecnología sino a dominarla y ponerla al servicio de todos.

\section{REFERENCIAS BIBLIOGRÁFICAS:}

BURBULES, N. C., CALLISTER, T. A.(h). Educación: riesgos y promesas de las nuevas tecnologías de la información. España. Ediciones Granica. 2001.

CASTELLS, M. La era de la información. Madrid. Alianza Editorial. 1997.

MAGGIO, M. "El tutor en la educación a distancia". En: Litwin, E. (compiladora): La educación a distancia. Colección Agenda Educativa. Buenos Aires. Amorrortu Editores S. A. 2000.

MAGLIO, F. M. (1998). "El positivismo y las ciencias sociales. ¿La concepción positivista de las Ciencias Sociales limita la posibilidad de comprender la realidad?".

http://www.didacticahistoria.com/ccss/ccss16.htm (Acceso: Agosto de 2002)

MATURANA, Humberto. (1997). Educar para colaborar o para competir, Revista de Pedagogía, pag, 133; Chile. http://www.mdnh.org/educacion/educadores.html\#4_3 (Acceso: Junio de 2002)

MATURANA, H. Conferencia en el V Congreso Internacional de Informática (RIBIE 2000), Chile.

O’CONNOR, Joseph; SEYMOUR, John. Introducción a la PNL. Barcelona. Ediciones Urano.1996. 
PEA, Roy D. "Prácticas de inteligencia distribuida y diseños para la educación". En: Libro Cogniciones distribuidas de Gabriel Salomon (compilador). Buenos Aires. Colección Agenda Educativa. Amorrortu Editores S. A.1993.

VILLASEÑOR SÁNCHEZ, G.. Conferencia en el V Congreso Internacional de Informática (RIBIE 2000), Chile. 2000. 